\title{
Über den Zündmechanismus einer fremdausgelösten Niederdruckfunkenstrecke mit Hilfsfunken in der Anode
}

\author{
W. Frie und A. Michel \\ Siemens Aktiengesellschaft, Forschungslaboratorium Erlangen \\ (Z. Naturforschg. 22 a, 1975-1980 [1967] ; eingegangen am 19. Juli 1967)
}

\begin{abstract}
Der Zündmechanismus einer getriggerten Niederdruckfunkenstrecke mit Hilfsgleitfunken in der Anode beruht im wesentlichen auf der Auslösung von Photoelektronen an der Kathode durch das von dem Hilfsfunken emittierte kurzwellige Licht. Eine Theorie dieses Vorganges wird entwickelt und mit den Meßergebnissen verglichen.
\end{abstract}

Niederdruckfunkenstrecken mit einer Halbleitergleitstrecke als Hilfsfunke in der Kathode sind in der Lage, Spannungen ab etwa $1 \mathrm{kV}$ mit sehr kleiner Verzögerungs- und Streuzeit wiederholt zu schalten ${ }^{1}$. Sie eignen sich deshalb auch für den Kurzschluß einer induktiven Last in der Umgebung des Strommaximums.

Der wesentliche Grund für dieses günstige Verhalten ist, daß der Halbleitergleitfunke in der Kathode während des Zündvorganges der Hauptentladung als hochergiebige Elektronenquelle dient, deren Raumladung in dem Maße momentan kompensiert wird, wie sich im Entladungsraum positive Ionen bilden. Der Triggerfunke allein hat eine sehr kurze Verzögerungs- und Streuzeit; die Streuzeit der Hauptentladung ist unmeßbar klein.

Wird der Triggerfunke in der Anode der Niederdruckfunkenstrecke angeordnet, so ist deren Verzögerungs- und Streuzeit um so größer, je kleiner die Zugspannung im Vergleich zur statischen Durchbruchspannung und je kleiner die dem Triggerfunken zugeführte Leistung ist. Über experimentelle Untersuchungen der Verzögerungs- und Streuzeit einer Niederdruckfunkenstrecke mit Trigger in der Anode und eine Theorie des Zündvorganges wird im folgenden berichtet.

\section{Versuchsanordnung}

Die verwendete Versuchsanordnung besteht im wesentlichen aus einer demontierbaren Niederdruckfunkenstrecke mit Wolfram-Kupfer-Elektroden $\left({ }^{1}, \mathrm{Abb} .1\right)$, die mit einem Impulskondensator von $4,5 \mu \mathrm{F}$ so zusammengebaut ist, daß sie den Kondensator bei Zündung direkt kurzschließt. Bei $20 \mathrm{kV}$ Ladespannung ergab sich ein Maximalstrom von $75 \mathrm{kA}$. Der Entladungsraum steht über den Pumpstutzen und eine Kühlfalle in flüssigem Stickstoff dauernd mit

1 W. Frie u. A. Michel, Z. Naturforschg. 22a, 1963 [1967]. einer Vorpumpe in Verbindung. Durch ein Nadelventil wird in die Funkenstrecke Luft eingelassen und der Druck mit einem Pirani-Manometer kontrolliert. Als Trigger dient eine demontierbare Koaxialstrecke (1, Abb. 2), bei der zwischen den Wolfram-Kupfer-Elektroden ein Plättchen aus Halbleitermaterial als Gleitfläche angebracht ist. Das Halbleiterplättchen bestand in der Regel aus Zink-Cadmium-Oxid ${ }^{2}$.

Drei verschiedene Triggerspannungskreise wurden verwendet. Ihre Daten sind in der folgenden Tabelle zusammengefaßt.

\begin{tabular}{|c|c|c|c|c|c|c|}
\hline & & $\begin{array}{l}L \\
\mathrm{H}\end{array}$ & $\begin{array}{l}C \\
\mathrm{~F}\end{array}$ & $\begin{array}{l}R \\
\Omega\end{array}$ & $\begin{array}{l}\text { Max. } \\
\text { Lade- } \\
\text { span- } \\
\text { nung } \\
\text { kV }\end{array}$ & Schaltelement \\
\hline 1 & 3,2 & $\cdot 10^{-6}$ & $1,25 \cdot 10^{-7}$ & 28 & 15 & $\begin{array}{l}\text { Thyratron } \\
\text { PL } 522\end{array}$ \\
\hline 2 & 2,09 & $9 \cdot 10^{-6}$ & $2,62 \cdot 10^{-7}$ & $4,1 \cdot 10$ & 20 & Hochdruck FS \\
\hline 3 & 3,27 & $7 \cdot 10^{-7}$ & $2,62 \cdot 10^{-7}$ & $9,6 \cdot 10^{-2}$ & 20 & Hochdruck FS \\
\hline
\end{tabular}

Als Zündverzögerung $t_{\mathrm{v}}$ wurde die Zeit zwischen dem Beginn des Zusammenbruchs der Triggerspannung und dem Fußpunkt der Wendetangente an die zeitliche Ableitung des Stromes durch die Niederdruckfunkenstrecke gemessen ( ${ }^{1}: t_{\mathrm{v}_{2}}$ in Abb. 7). Die Beobachtung des Zündverzuges erfolgte visuell auf einem Zweistrahloszillographen (28 MHz Bandbreite), der mit einem Strahl die Triggerspannung und mit dem anderen die Stromanstiegsgeschwindigkeit registrierte.

\section{Versuchsergebnisse}

Abb. 1 und 2 zeigen die aus je 100 Versuchen ermittelte Verteilung der Zündverzüge bei Anordnung der Triggerfunkenstrecke in der Anode für 2,5 $10^{-2}$ Torr Luft mit der Zugspannung und der Triggerleistung als Parameter. In halblogarithmischer Darstellung ist über der Zeit $t$ der Bruchteil $N / N_{0}$ von $N_{0}$ Zündversuchen aufgetragen, bei dem der Verzug größer als $t$ ist.

2 DBP 1191450. 


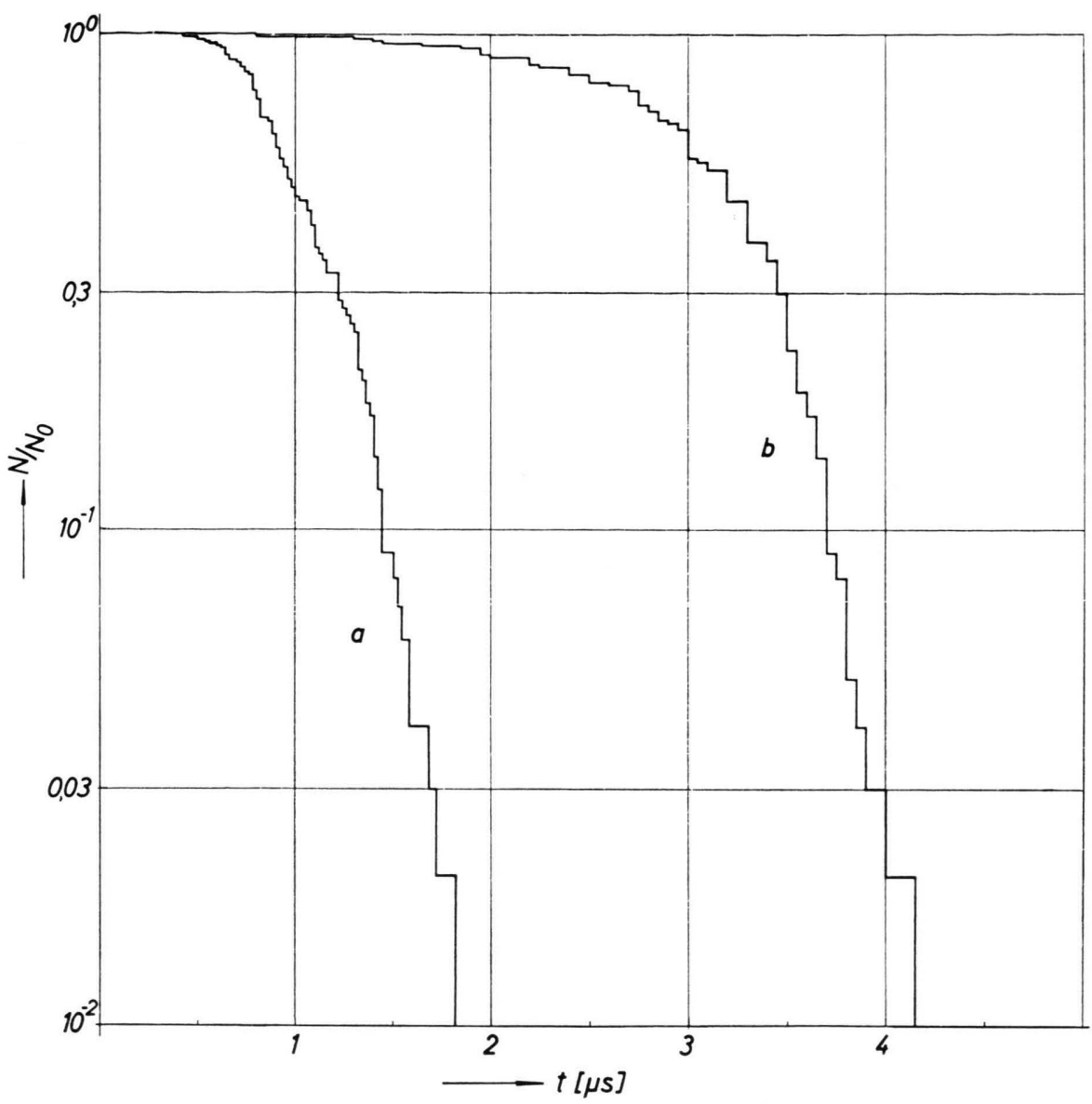

Abb. 1.Verteilung der Zündverzüge einer Niederdruckfunkenstrecke mit Trigger in der Anode. Trigger-Kondensator-Ladespannung 15 $\mathrm{kV}$, feldverstärkend. $p_{0}=$ $2,5 \cdot 10^{-2}$ Torr. a Zugspannung $20 \mathrm{kV}$, b Zugspannung $5 \mathrm{kV}$.

Abb. 1 gibt die Verteilung bei 5 und $20 \mathrm{kV}$ Zugspannung wieder. Als Triggerspannungskreis wurde ein Thyratronkreis (Nr. 1 der Tabelle) verwendet, der einen positiven, nahezu kritisch gedämpften $15 \mathrm{kV}$-Impuls erzeugte.

Abb. 2 zeigt die Verteilung bei $20 \mathrm{kV}$ Zugspannung und sehr schwach gedämpftem positivem Triggerimpuls, der von den $20 \mathrm{kV}$-Funkenstreckenkreisen Nr. 2 bzw. 3 der Tabelle erzeugt wurde.

Die Triggerspannungsgeneratoren unterscheiden sich dadurch, daß sie in der Reihenfolge der Nummerierung durch Vergrößerung der Kapazität und Verkleinerung von Widerstand und Selbstinduktion des Entladungskreises immer größere Impulsleistung abgeben.

Die Verteilungskurven in Abb. 1 zeigen zunächst, da $\beta$ bei gleicher mittlerer Triggerleistung mit zunehmender Zugspannung die kürzeste Verzögerungsund die mittlere Streuzeit kleiner werden. Aus Abb. 2 ergibt sich, daß diese Zeiten bei konstanter Zug-

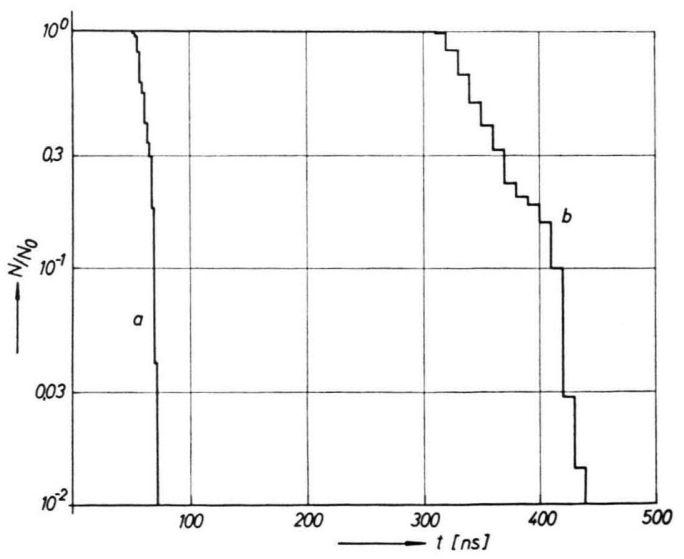

Abb. 2. Verteilung der Zündverzüge einer Niederdruckfunkenstrecke mit Trigger in der Anode. Trigger-Kondensator-Ladespannung $20 \mathrm{kV}$, feldverstärkend. $p_{0}=2,5 \cdot 10^{-2}$ Torr. Zugspannung $20 \mathrm{kV}$. Induktivität des Triggerkreises: a $L=3,3 \cdot 10^{-7} \mathrm{H}$, b $L=2,1 \cdot 10^{-6} \mathrm{H}$.

spannung mit zunehmender Triggerleistung ebenfalls kleiner werden. Im Hinblick auf den Einfluß 
der Zugspannung und der Triggerleistung auf die Funkenverzögerung verhält sich also die Niederdruckstrecke mit Trigger in der Anode ähnlich wie eine Hochdruckstrecke. Allen Kurven gemeinsam ist mit Ausnahme der Abb. $2 \mathrm{~b}$ der gekrümmte $\mathrm{Ab}$ fall von der Horizontalen $N / N_{0}=1$ und der Knick im Bereich von 0,1-0,3. Bei Abb. 1 b scheint dieser Knick mit der Anfangskrümmung zusammenzufallen. Die Anfangskrümmung ist nach FELDT und RAETHER $^{3,4}$ charakteristisch für eine sehr hohe Primärelektronenerzeugungsrate durch Fremdeinstrahlung. Unter dieser Bedingung strebt die Wahrscheinlichkeit, vor der Kathode ein ,Startelektron“ innerhalb $\mathrm{d} t$ anzutreffen, gegen Eins und die Streuung gegen Null. Als Folge davon macht sich eine Streuung der Aufbauzeit bemerkbar, die durch Schwankungen in der kathodenseitigen Elektronennachlieferung während des Lawinenablaufs verursacht wird. Die Lauesche Statistik ist durch die Auf baustatistik nach FeLdT, RAETHER und Legler zu ersetzen.

\section{Theorie des Zündvorganges}

Bei einer fremdausgelösten Funkenstrecke mit Trigger in der Anode und feldverstärkender Triggerspannung liegt es zunächst nahe, für den Zündmechanismus einen Stoßdurchschlag anzunehmen. Nun erreicht aber die Spannung am Triggerstift der Halbleiterstrecke abhängig von der Induktivität des Triggerkreises nur maximal einige Kilovolt. Die Summe aus Zugspannung und maximaler Spannung am Triggerstift ist kleiner als die statische Durchbruchspannung zwischen Triggerstift und Kathode. Sie reicht also selbst bei feldverstärkender Triggerspannung nicht aus, um einen Stoßdurchschlag herbeizuführen. Ein anderer Mechanismus muß die Zündung der Niederdruckfunkenstrecke bewirken.

Es ist bekannt, daß ein Funke sehr kurzwelliges Licht aussendet. Befindet sich die Gleitfunkenstrecke in der Anode, so werden durch Bestrahlung der Kathode Photoelektronen ausgelöst und von der Zugspannung abgesaugt. Der Strom ist raumladungsbegrenzt. Auf dem Weg zur Anode stoßen die Elektronen mit Neutralgasmolekülen zusammen, die dadurch teilweise ionisiert werden. Der Strom steigt mit der Zeit an und die Strecke zündet, wenn eine kritische Stromstärke erreicht ist.

3 W. Feldt u. H. Raether: Ann. Phys. Leipzig 18, 370 [1956].

4 W. Legler, Ann. Phys. Leipzig 18, 374 [1956].
Während des Zündvorganges sei angenommen, da $\beta$ die Trägerverluste vernachlässigbar sind und die Potentialverteilung in erster Näherung zeitunabhängig ist. Wird der Weg $x$ ab der Kathode in Richtung auf die Anode $(x=d)$ gezählt, so gilt für die Bilanz der negativen Träger:

$$
\frac{\partial n_{\mathrm{e}}}{\partial t}+\frac{\partial\left(n_{\mathrm{e}} v\right)}{\partial x}=n_{\mathrm{e}} n_{0} q v .
$$

Hier und im folgenden bedeuten:

$n_{\mathrm{e}}=$ Elektronendichte,

$n_{0}=$ Neutralteilchendichte,

$q=$ Wirkungsquerschnitt für Ionisation durch Elektronenstoß,

$v=$ Elektronengeschwindigkeit,

$j_{\mathrm{e}}=$ Elektronenstromdichte,

$j_{\Phi}=$ Photostromdichte herrührend von der Triggerentladung,

$i_{\mathrm{TE}}=$ Triggerentladungsstrom,

$U_{0}=$ Ladespannung des Triggerkondensators,

$L=$ Induktivität des Triggerkreises.

Mit

folgt

$$
j_{\mathrm{e}}=n_{\mathrm{e}} e v, \quad v=v(x), \quad q=q(v(x))
$$

$$
\begin{aligned}
\frac{\partial j_{\mathrm{e}}}{\partial t}+v(x) \frac{\partial j_{\mathrm{e}}}{\partial x} & =j_{\mathrm{e}} n_{0} q(x) \cdot v(x) \\
& =j_{\mathrm{e}} \alpha(x) \cdot v(x) .
\end{aligned}
$$

$v(x)$ ist der Mittelwert der Elektronengeschwindigkeit an der Stelle $x$. Er ist bei niedrigem Gasdruck wegen der kleinen Stoßzahl praktisch gleich der Freifluggeschwindigkeit.

Der Ansatz

$$
\begin{aligned}
j_{\mathrm{e}}(x, t) & =\exp \left(\int_{0}^{x} \alpha(x) \mathrm{d} x\right) f(z), \\
z & =t-\int_{0}^{x}(1 / v(x)) \mathrm{d} x
\end{aligned}
$$

ist mit beliebigem $f(z)$ eine Lösung von (1a). Als Abkürzung sei eingeführt:

$$
I_{\alpha}=\int_{0}^{d} \alpha(x) \mathrm{d} x, \quad I_{v}=\int_{0}^{d}(1 / v(x)) \mathrm{d} x .(4 \mathrm{a}, \mathrm{b})
$$

Zur Bestimmung von $f(z)$ wird angenommen, daß Elektronen an der Kathode nur durch kurzwelliges Licht ${ }^{5,6}$ ausgelöst werden, welches einerseits von der Entladung selbst bei Elektronenstoßanregung der Gasmoleküle sowie bei Abbremsung der Entladungselektronen an der Anode und andererseits von dem Triggerfunken emittiert wird. Weiterhin

5 L. B. Loeb, Rev. Mod. Phys. 8, 267 [1926].

6 W. Bartholomeyczyk, Z. Phys. 116, 235 [1940]. 
soll die Raumladungsbegrenzung des aus der Kathode austretenden Elektronenstromes in dem Maße aufgehoben werden, wie sich im Entladungsraum positive Ionen bilden ${ }^{1,7}$. Somit gilt:

$$
\begin{aligned}
j_{\mathrm{e}}(x=0, t) & =\int_{0}^{d} C^{\prime}(x) j_{\mathrm{e}}(x, t) d x+j_{\Phi}(t) \\
& +\int_{0}^{d} C^{\prime \prime}(x)\left\{j_{\mathrm{e}}(x, t)-j_{\mathrm{e}}(x=0, t=0)\right\} \mathrm{d} x \\
& +j_{\mathrm{e}}(x=0, t=0) .
\end{aligned}
$$

Mit

$$
j_{\mathrm{e}}(x=0, t=0)=0
$$

folgt :

$$
j_{\mathrm{e}}(x=0, t)=\int_{0}^{d} C(x) j_{\mathrm{e}}(x, t) \mathrm{d} x+j_{\Phi}(t)
$$

mit

$$
C(x)=C^{\prime}(x)+C^{\prime \prime}(x) .
$$

Ist $C(x)$ im Intervall $0 \leqq x \leqq d$ integrierbar und $j_{\mathrm{e}}(x, t)$ dort monoton, so gibt es einen innerhalb des Intervalls liegenden festen Abstand $\xi$, für den gilt:

$$
\begin{aligned}
\int_{0}^{d} C(x) j_{\mathrm{e}}(x, t) \mathrm{d} x= & j_{\mathrm{e}}(x=0, t) \int_{0}^{\xi} C(x) \mathrm{d} x \\
& +j_{\mathrm{e}}(x=d, t) \int_{\xi}^{d} C(x) \mathrm{d} x,
\end{aligned}
$$

also :

$$
\begin{aligned}
j_{\mathrm{e}}(x=0, t)= & \frac{\int_{\xi}^{d} C(x) \mathrm{d} x}{1-\int_{0}^{\xi} C(x) \mathrm{d} x} \cdot j_{\mathrm{e}}(x=d, t) \\
& +\frac{1}{1-\int_{0}^{\xi} C(x) \mathrm{d} x} \cdot j_{\Phi}(t) .
\end{aligned}
$$

Die Intensität des von einem Niederdruckgleitfunken emittierten weichen RöNTGEN-Lichtes ist in guter Näherung dem Quadrat des Gleitfunkenstromes proportional ${ }^{8}$.

$$
j_{\Phi}(t)=C_{3} i_{\mathrm{TE}}^{2}(t) .
$$

Steigt der Triggerstrom linear mit der Zeit an, wie es zu Beginn einer Kondensatorentladung über eine Induktivität näherungsweise der Fall ist, so wird:

$$
j_{\Phi}(t)=C_{3}\left(U_{0} / L\right)^{2} t^{2} .
$$

7 R. Hancox, Rev. Sci. Instr. 33, 1239 [1962].

8 P. Bogen u. H. Conrads, Proc. VIth Conf. on Ionization Phenomena in Gases, Paris 1963, III/337.
Somit :

$$
\begin{aligned}
j_{\mathrm{e}}(x=0, t)= & \frac{\int_{\xi}^{d} C(x) \mathrm{d} x}{1-\int_{0}^{\xi} C(x) \mathrm{d} x} j_{\mathrm{e}}(x=d, t) \\
& +\frac{C_{3} U_{0}{ }^{2}}{\left.\mid 1-\int_{0}^{\xi} C(x) \mathrm{d} x\right] \cdot L^{2}} \cdot t^{2} \\
= & C_{1} j_{\mathrm{e}}(x=d, t)+C_{2} t^{2}
\end{aligned}
$$

mit den Nebenbedingungen:

$$
\begin{gathered}
j_{\mathrm{e}}(x=0, t=0)=0, \\
j_{\text {gesamt }}(t)=j_{\mathrm{e}}(x=d, t) .
\end{gathered}
$$

Wird (2) in (13a) eingesetzt, so folgt:

$$
f(t)=C_{1} \exp \left(I_{\alpha}\right) f\left(t-I_{v}\right)+C_{2} t^{2} .
$$

Diese Gleichung ist eine inhomogene Differenzengleichung, deren Lösung sich aus einem homogenen und einem inhomogenen Anteil zusammensetzt. Mit dem Ansatz:

$$
\begin{aligned}
f(z)= & A \cdot \exp (z / \tau) \\
& -C_{2} \tau^{2}\left[a_{0}+a_{1}(z / \tau)+a_{2}(z / \tau)^{2}\right]
\end{aligned}
$$

folgt für den homogenen Teil durch Einsetzen:

$$
\begin{aligned}
\exp \left(I_{v} / \tau\right) & =C_{1} \exp \left(I_{\alpha}\right), \\
\tau & =I_{v} /\left(I_{\alpha}+\ln C_{1}\right)
\end{aligned}
$$

und für den inhomogenen Anteil:

$$
\begin{aligned}
a_{0}+ & a_{1}(t / \tau)+a_{2}(t / \tau)^{2} \\
= & \exp \left(I_{v} / \tau\right)\left\{a_{0}+a_{1}\left(t-I_{v}\right) / \tau+a_{2}\left[\left(t-I_{v}\right) / \tau\right]^{2}\right\} \\
& -(t / \tau)^{2} .
\end{aligned}
$$

Die Koeffizienten ergeben sich durch Koeffizientenvergleich und hängen nur von $I_{v} / \tau$ ab. Die Konstante $A$ bestimmt sich aus der Anfangsbedingung (6) zu:

$$
A=C_{2} \tau^{2} a_{0} .
$$

Für große Zeiten kann der inhomogene gegenüber dem homogenen Anteil der Lösung vernachlässigt werden und es folgt wegen der Randbedingung (14) im Rahmen der Näherung:

$$
\begin{aligned}
j_{\text {gesamt }}(t) & =\exp \left(I_{\alpha}\right) a_{0} C_{2} \tau^{2} \exp (t / \tau) \exp \left(-I_{v} / \tau\right) \\
& =\left(a_{0} C_{2} \tau^{2} / C_{1}\right) \exp (t / \tau) .
\end{aligned}
$$


Wegen (11) ist, solange der Triggerstrom zeitproportional ansteigt:

$$
\begin{aligned}
C_{2} & =\frac{C_{3}}{1-\int_{0}^{\xi} C(x) \mathrm{d} x}\left[\frac{i_{\mathrm{TE}}(t)}{t}\right]^{2} \\
& =C_{4}\left[\frac{i_{\mathrm{TE}}(t)}{t}\right]^{2} .
\end{aligned}
$$

Somit wird :

$$
j_{\text {gesamt }}(t)=\left(a_{0} C_{4} / C_{1}\right)(\tau / t)^{2} i_{\mathrm{TE}}^{2}(t) \exp (t / \tau) .
$$

Zur Zeit $t_{\mathrm{v}}$ erreicht die Stromdichte einen kritischen festen Wert $j_{\mathrm{k}}$, und die Funkenstrecke zündet.

$$
j_{\text {gesamt }}\left(t_{\mathrm{v}}\right)=j_{\mathrm{k}},
$$

$\ln j_{\mathrm{k}}=\ln \left(\frac{a_{0} C_{4}}{C_{1}}\right)+2\left[\ln i_{\mathrm{TE}}\left(t_{\mathrm{v}}\right)+\ln \frac{\tau}{t_{\mathrm{v}}}\right]+\frac{t_{\mathrm{v}}}{\tau}$.

Somit ist :

$$
\frac{\mathrm{d} \ln i_{\mathrm{TE}}\left(t_{\mathrm{v}}\right)}{\mathrm{d} t_{\mathrm{v}}}=1 / t_{\mathrm{v}}-1 /(2 \tau)
$$

oder:

$$
\frac{\mathrm{d} t_{\mathrm{v}}}{\mathrm{d} \ln i_{\mathrm{TE}}\left(t_{\mathrm{v}}\right)}=\frac{2 \tau t_{\mathrm{v}}}{2 \tau-t_{\mathrm{v}}} \approx-2 \tau \text { für } t_{\mathrm{v}} \gg \tau
$$

In (13) ist:

$$
C_{2}=\frac{C_{3} U_{0}^{2}}{\left[1-\int_{0}^{\xi} C(x) \mathrm{d} x\right] L^{2}}=\frac{C^{5}}{L^{2}} .
$$

Somit wird :

$$
\begin{array}{r}
\ln j_{\mathrm{k}}=\ln \left(a_{0} C_{5} / C_{1}\right)+2(\ln \tau-\ln L]+t_{\mathrm{v}} / \tau \\
\text { und } \frac{\mathrm{d} \ln L}{\mathrm{~d} t_{\mathrm{v}}}=\frac{1}{2 \tau} \quad(29) \quad \text { oder } \quad \frac{\mathrm{d} t_{\mathrm{v}}}{\mathrm{d} \ln L}=2 \tau .
\end{array}
$$

\section{Diskussion}

Die an einer Niederdruckfunkenstrecke mit aufgesprühten Wolfram-Elektroden bei $2 \cdot 10^{-2}$ Torr und $20 \mathrm{kV}$ Zugspannung gemessene mittlere Verzögerungszeit $t_{\mathrm{v}}$ ist in Abb. 3 als Funktion des Logarithmus des Triggerstromes $i_{\mathrm{TE}}\left(t_{\mathrm{v}}\right)$ zur Zeit $t_{\mathrm{v}}$ und in Abb. 4 als Funktion des Logarithmus der Triggerkreisinduktivität $L$ aufgetragen. Der Triggerkreisstrom stieg während der gemessenen Verzögerungszeiten linear mit der Zeit an. Die Triggerkreiskapazität (20 kV Ladespannung) betrug $2,59 \cdot 10^{-7} \mathrm{~F}$. Der Verlauf der gemessenen Verzögerungszeiten ist in Übereinstimmung mit der Theorie. Abb. 3 zeigt einen anfänglich schwach gekrümmten Verlauf, der entsprechend (26) mit $t_{\mathrm{v}} \rightarrow 2 \tau$ in einen stärker gekrümmten Abschnitt mit annähernd vertikaler Tangente übergeht. Abb. 4 ist entsprechend (30) eine

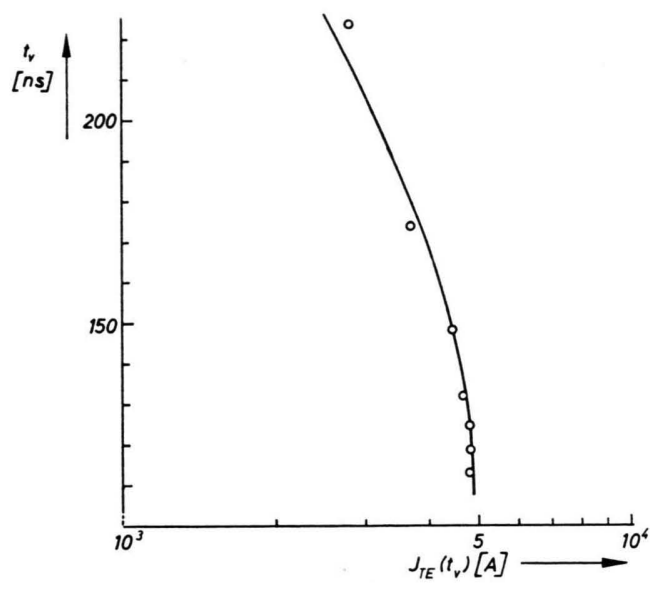

Abb. 3. Mittlere Verzögerungszeit $\overline{t_{\mathrm{v}}}$ einer Niederdruckfunkenstrecke mit Trigger in der Anode als Funktion des Triggerstromes zur Zeit $\overline{t_{\mathrm{v}}} . \quad p_{0}=2 \cdot 10^{-2}$ Torr. Zugspannung $20 \mathrm{kV}$.

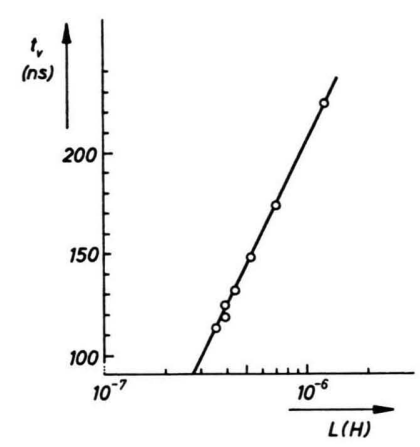

Abb. 4. Mittlere Verzögerungszeit einer Niederdruckfunkenstrecke mit Trigger in der Anode als Funktion der Induktivität des Triggerkreises. $p_{0}=2 \cdot 10^{-2}$ Torr. Zugspannung $20 \mathrm{kV}$. Ladespannung des Triggerkondensators $+20 \mathrm{kV}$.

halblogarithmische Gerade, aus deren Neigung sich eine Zeitkonstante von $4,5 \cdot 10^{-8}$ sec ergibt. Da in dem Ausdruck für die Zeitkonstante (17a) nur die Elektronen- und nicht die Ionengeschwindigkeit auftritt, vermag die Theorie auch die experimentell gefundenen kleinen Verzögerungszeiten befriedigend zu erklären.

Wird die Polarität der Triggerspannungswelle so gewählt, daß sie zu Beginn des Schaltvorganges schwächend auf das elektrische Feld in der Funkenstrecke wirkt, so ist ein Stoßdurchschlagseinfluß auf die Verteilung der Zündverzüge ausgeschlossen. In Abb. 5 ist eine mit feldschwächender Triggerspannung erhaltene Verteilung zusammen mit der Verteilung aus Abb. 2a wiedergegeben, die bei Feldverstärkung aber unter sonst gleichen Bedingungen 


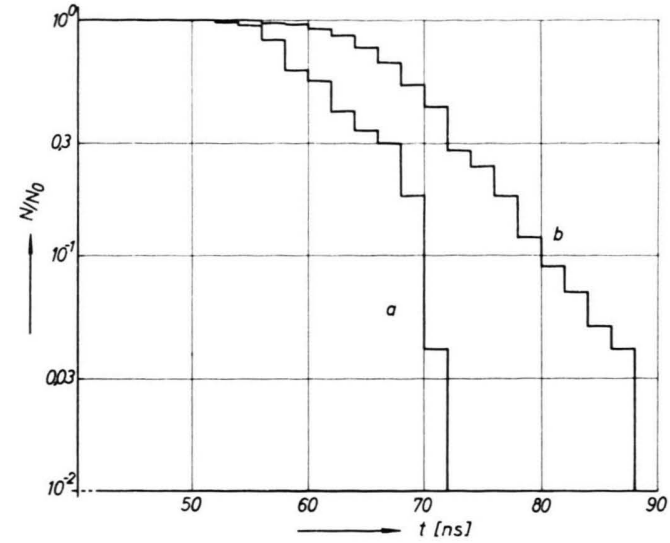

Abb. 5. Verteilung der Zündverzüge einer Niederdruckfunkenstrecke mit Trigger in der Anode. $p_{0}=2,5 \cdot 10^{-2}$ Torr. Zugspannung $20 \mathrm{kV}$. Induktivität des Triggerkreises $3,3 \cdot 10^{-7} \mathrm{H}$. Triggerkondensator-Ladespannung $20 \mathrm{kV}$; Polarität: a feldverstärkend (identisch mit Abb. 2a), b feldschwächend. aufgenommen wurde. Die Kurven unterscheiden sich in der kürzesten Aufbauzeit und der mittleren Streuzeit; weiterhin zeigt die Verteilung bei feldverstärkender Triggerspannung den bereits erwähnten Knick bei $N / N_{0} \approx 0,3$, der bei Feldschwächung nicht auftritt.

Der Knick, die geringfügig verkürzte kleinste Aufbauzeit und die von 14 auf 10 ns reduzierte mittlere Streuzeit lassen sich somit auf die Auswirkung der Feldverstärkung durch die Triggerspannung zurückführen.

Der Einfluß der Feldverstärkung auf die Verteilung der Zündverzüge ist gering, da die Spannung an der gezündeten Triggerfunkenstrecke nur die Größenordnung $1 \mathrm{kV}$ erreicht.

Fräulein D. Schwab sowie den Herren J. Stroh und F. BECKER sei für ihre Mithilfe bei den Experimenten, Herrn W. Hertz für wertvolle Diskussionen herzlich gedankt.

\title{
Über die Zündwahrscheinlichkeit einer Niederdruckfunkenstrecke bei Spannungswiederkehr nach Strombelastung
}

\author{
W. Frie und A. Michel \\ Siemens Aktiengesellschaft, Forschungslaboratorium Erlangen \\ (Z. Naturforschg. 22 a, 1980-1984 [1967] ; eingegangen am 26. Juli 1967)
}

\begin{abstract}
Kehrt bei einer fremdauslösbaren Niederdruckfunkenstrecke nach vorangegangener Impulsstrombelastung die Elektrodenspannung zeitverzögert wieder, so nimmt die Wahrscheinlichkeit der Selbstzündung mit fortschreitender Entionisierung ab. Die Durchbruchshäufigkeit als Funktion der Verzögerungszeit beschreibt die Wiederkehr der Spannungsfestigkeit. Eine von der Entionisierung durch ambipolare Diffusion und Volumenrekombination ausgehende Theorie der Wiederzündwahrscheinlichkeit ist in guter Ưbereinstimmung mit dem Experiment. Sie gilt auch für die den gleichen Mechanismen unterliegenden Ignitrons und Vakuum-Leistungsschalter.
\end{abstract}

Bei fremdausgelösten Niederdruckfunkenstrecken mit Hilfsfunken in Kathode ${ }^{1}$ oder Anode ${ }^{2}$ ist nicht nur das Schaltvermögen und der Zündmechanismus von Interesse, sondern auch die Frage, wie schnell die Strecke nach vorangehender Strombelastung wieder spannungsfest wird. An die Elektroden kann nach Ende des Stromflusses nicht sofort wieder die volle Arbeitsspannung angelegt werden, da die Leitfähigkeit des Plasmas nur langsam abklingt. Die Zeit bis zur Wiederkehr der Spannungsfestigkeit wird abhängig sein von der Art und dem Druck des Füllgases, dem Strom, der Stromflußdauer und den Elektroden- und Wandmaterialien des Entladungs-

1 W. Frie u. A. Michel, Z. Naturforschg. 22a, 1963 [1967]. gefäßes. Weiter kann die Expansionsmöglichkeit für das Entladungsplasma und die Sauggeschwindigkeit der Vakuumpumpe einen Einfluß haben.

\section{Experimentelle Anordnung}

Um die Wiederkehr der Spannungsfestigkeit zu messen, ist ein einmaliger Rechteckstromstoß zur Ionisation des Gases am günstigsten. Ein solcher Verlauf ist aber experimentell nur mit großem Aufwand herzustellen, und es wurden deshalb nahezu kritisch gedämpfte Stromimpulse verwendet. Die Meßschaltung ist in Abb. 1 wiedergegeben. Nicht

2 W. Frie u. A. Michel, Z. Naturforschg. 22a, 1975 [1967]. 